\section{Regression nach Passing-Bablok}

R.-D. Hilgers ${ }^{1}$, N. Heussen ${ }^{1}$ und S. Stanzel ${ }^{2}$

${ }^{1}$ Institut für Medizinische Statistik, Universitätsklinikum der RWTH Aachen, Aachen, Deutschland

${ }^{2}$ DKFZ Heidelberg, Heidelberg, Deutschland

Englischer Begriff Passing-Bablok regression

Definition Die Regression nach Passing-Bablok ist ein spezielles Verfahren zur Testung der Gleichheit von Messungen zweier unterschiedlicher analytischer Methoden.

Beschreibung Die Regression nach Passing-Bablok ist ein Verfahren zum Nachweis der Gleichheit von Messungen (s. > Messung) zweier unterschiedlicher analytischer Methoden, das keine Verteilungsannahmen an Einzelbeobachtungen und Messfehler ( $\triangleright$ Messgenauigkeit) macht. Es handelt sich dabei um ein lineares Regressionsverfahren ( $\triangleright$ Regression, lineare), bei dem die $\triangleright$ Schätzer für den $\triangleright$ Achsenabschnitt und die Steigung ( $\triangleright$ Regressionskoeffizient) der Regressionsgeraden (s. $>$ Regressionsgerade) über die Berechnung des Medians ( $\triangleright$ Median) der Steigungsdreiecke aller möglichen Messwertpaare ermittelt werden. Anschließend wird die Gleichheit der Messungen der beiden Messmethoden durch 2 Hypothesentests ( $\triangleright$ Test, statistischer) überprüft. Werden die $\triangleright$ Nullhypothesen beider Tests (Test A: Achsenabschnitt $=0$; Test B: Steigung $=1$ ) nicht verworfen, kann auf die Gleichheit der Messungen beider Messmethoden geschlossen werden.

\section{Literatur}

Passing H, Bablok W (1983) A new biometrical procedure for testing the equality of measurements from two different analytical methods. Application of linear regression procedures for method comparison studies in clinical chemistry, part I. J Clin Chem Clin Biochem 21:709-720 